DOI: https://doi.org/10.34069/AI/2021.46.10.14

How to Cite:

Pavliuk, M., Hrys, A., Serhieienkova, O., Nekiz, T., \& Yatsyna, O. (2021). Actitud valorativa de la juventud estudiantil hacia el estilo de vida saludable y la actividad física como factores que contribuyen a su éxito. Amazonia Investiga, 10(46), 152-161. https://doi.org/10.34069/AI/2021.46.10.14

\title{
Actitud valorativa de la juventud estudiantil hacia el estilo de vida saludable y la actividad física como factores que contribuyen a su éxito
}

\section{Value-based attitude of student youth towards a healthy lifestyle and physical activity as factors of their aspiration for success}

Received: October 1, 2021

Accepted: November 2, 2021

\section{Resumen}

El objetivo del estudio empírico es determinar la actitud valorativa de la juventud estudiantil hacia el estilo de vida saludable en particular la actividad física y la influencia en los parámetros de autorregulación voluntaria del comportamiento de estudiantes y su deseo de tener éxito. El estudio empírico se organizó y se realizó entre los encuestados de las facultades que no están relacionadas con la educación física profesional ni el deporte. Materiales y métodos. Métodos: pruebas con cuestionarios estandarizados. Resultados. Se estableció una relación estadísticamente significativa entre los parámetros de autorregulación de los encuestados: "Independencia", "Evaluación de resultados", "Planificación", "Flexibilidad" y la necesidad de éxito ( $p<.01)$. Se encontraron las diferencias significativas entre los encuestados que tienen diferentes niveles de estilo de vida saludable según los parámetros de autorregulación del comportamiento $\square$ "Evaluación de los resultados" ( $p<.05)$,

\begin{abstract}
The purpose of the empirical research is to determine students' value-based attitude towards a healthy lifestyle, in particular, physical activity and its impact on parameters of volitional selfregulation of students' behavior and their aspiration for success. The empirical research was organized and conducted to examine the respondents taking courses at the faculties not related to professional training in physical education and sport. Materials and methods. Methods: tests with standardized questionnaires. Results. The study determines a statistically significant correlation between the parameters of the respondents' self-regulation: "Independence", "Evaluation of results", "Planning", "Flexibility" and the need to achieve success $(p<.01)$. The research found essential differences between the respondents with a different level of following a healthy lifestyle by the parameters of behavioral self-regulation "Evaluation of results" $(\mathrm{p}<.05)$, "Planning" $(\mathrm{p}<$ $.05)$, "Independence" $(\mathrm{p}<.05)$ and "Flexibility"
\end{abstract}

\footnotetext{
${ }^{50}$ Doctor of Psychological Sciences, Full Professor of the Department of Psychology, Interregional Academy of Personnel Management, Kyiv, Ukraine.

${ }^{51}$ Doctor of Psychological Sciences, Full Professor, Head of the Laboratory of Psychology of Socially Maladjusted Juveniles, Kostiuk Institute of Psychology of the NAPS of Ukraine, Kyiv, Ukraine.

${ }^{52}$ Doctor of Psychological Sciences, Full Professor of the Department of Psychology and Social Practices Department, Institute of Human Sciences, Borys Grinchenko Kyiv University, Kyiv, Ukraine.

${ }^{53}$ Postgraduate Student of the Department of Psychology, National Pedagogical Drahomanov University, Kyiv, Ukraine.

${ }^{54}$ Doctor of Pedagogical Sciences, Associate Professor, Professor of the Department of Health Sciences, Uzhhorod National University, Uzhgorod, Ukraine.
} 


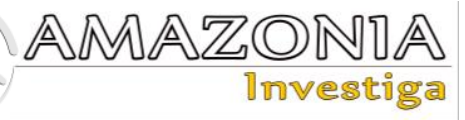

"Planificación" ( $\mathrm{p}<.05)$, "Independencia" ( $\mathrm{p}<$ $.05)$ y "Flexibilidad" $(\mathrm{P}<.05)$. Conclusiones. Se argumentó que la conciencia del estilo de vida saludable, de la actividad física y la recreación activa es un valor que influye en la autorregulación de los estudiantes, en su actividad volitiva y tiene un efecto positivo en la aspiración del éxito de la juventud estudiantil.

Palabras clave: estudiante, socialización de la juventud, motivación para el éxito, acto volitivo, autorregulación del comportamiento.

\section{Introducción}

El estilo de vida saludable, la actividad física son fenómenos importantes de la vida moderna, que no solo reflejan el sistema de valores de la sociedad, sino que afectan también la socialización de los jóvenes, crean un sistema de valores en los que se enfocan los estudiantes, forman la imagen y el estilo de vida.

Los estudios demuestran que los jóvenes que llevan la vida saludable, practican deportes, se adaptan mejor al entorno en el contexto social y psicológico que los que se quedan indiferentes ante la actividad física. La capacidad y el interés hacia los deportes tienen un efecto positivo para el desarrollo del conocimiento de sí mismo y también aumentan el prestigio entre los compañeros (Bugulov \& Sokaev, 2018; Byhovskaja, 2010). Cabe señalar que a los mecanismos que contribuyen al desarrollo de la motivación individual hacia el estilo de vida saludable, el deporte, se debe atribuir la identificación con la imagen de una persona sana que practica deporte.

La actividad deportiva cultiva valores sociales importantes como los de vencimiento, de justicia, de honestidad. Practicando deportes, o incluso observando las competiciones deportivas, el individuo asimila los valores sociales que se interiorizan en valores personales (Vnukov, 2014).

La actividad física tiene un efecto positivo desde el punto de vista de ideales y valores humanísticos. Cabe destacar su papel importante para el mantenimiento y fortalecimiento de la salud humana, el mejoramiento físico, la estimulación de la actividad, el deseo de desarrollar diversas habilidades, talentos $\mathrm{y}$ destrezas, adquirir nuevos conocimientos, establecer nuevos contactos productivos.

Últimamente los científicos están examinando la actividad física y el deporte desde el punto de $(\mathrm{p}<.05)$. Conclusions. The study substantiates that awareness of a healthy lifestyle, sporting activities and active leisure affects the students' self-regulation, volitional activity and has a positive effect on the student youth's aspiration for success.

Key words: student, youth socialization, motivation for success, volitional act, behavioral self-regulation.

vista de su potencial social y valorativo. Los valores sociales y personales de la cultura física y los deportes forman una parte importante de la cultura general. Hay que prestar atención a los valores personales, que determinan las actividades del individuo, orientado hacia los valores del estilo de vida saludable y el deporte que, a su vez, caracterizan el mundo interior del individuo y determinan las características estables, inmutables y absolutas del ser humano (Byhovskaja, 2010).

Es importante también tener en cuenta el potencial valorativo de la actividad física y el deporte lo que permite resolver una serie de problemas educativos. Por ejemplo, las actividades deportivas ayudan a los jóvenes deportistas a superar situaciones difíciles de la vida que aparecen en su entorno social (colegio, grupo de estudiantes, familia). Los atletas señalan que el deporte les ayudó a convertirse en una persona que tiene sólidos principios de vida, a ser independientes y decididos. En el proceso de la actividad deportiva ellos se dan cuenta de que el éxito depende solo de ellos: de su trabajo, paciencia, voluntad, perseverancia. La particularidad valorativa del deporte corresponde a las necesidades sociales de la sociedad (Burcev et al., 2014).

El estilo de vida saludable y la cultura física no sirven sólo para fortalecer la salud del estudiante, mejorar su estado físico, aumentar la actividad social, sino que también afectan significativamente otros aspectos de la vida humana: la actividad profesional, cualidades laborales, morales e intelectuales; influye en el proceso del autoconocimiento y superación personal, en la formación de la voluntad, etc. En la estructura de la cultura de la personalidad moderna, el estilo de vida saludable juega un papel importante y tiene el sentido del valor para el desarrollo armonioso. 
Como parte de la cultura, el estilo de vida saludable y la actividad física se relacionan con las categorías valorativas que acumulan y transmiten: 1) afectan directamente el desarrollo físico y espiritual del individuo, la divulgación del potencial creativo de los participantes del proceso deportivo; 2) son el medio de formar el modo y estilo de vida, son un ejemplo cultural del impacto positivo en la conciencia de personas, en las relaciones entre ellas; 3) demuestran una variedad de ejemplos y modelos del comportamiento individual que corresponden al máximo a las características particulares de cada individuo; 4) son un instrumento de influencia en el desarrollo físico, intelectual y espiritual de la juventud; 5) son un modelo de interacción social que se basa en el principio democrático de la justicia y la igualdad de oportunidades; 6) son un medio de comunicación universal y contribuyen al desarrollo de contactos interpersonales e internacionales. Los modelos simbólicos, los ejemplos culturales y los sentidos relacionados con la cultura física y el deporte se actualizan en la sociedad que se centra en los valores del desarrollo sostenible (Bugulov \& Sokaev, 2018).

Examinando el potencial valorativo de la cultura física de estudiantes, podemos distinguir dos niveles de valores: personales y sociales. El nivel personal de la asimilación de valores de la educación física está determinado por los conocimientos de los estudiantes como mejorarse físicamente, por las habilidades motoras, la capacidad de autorregulación del estilo de vida saludable, las actitudes sociales psicológicas, la orientación hacia el deporte y la actividad física. Cuando los estudiantes asimilan los valores sociales de la educación física como parte integral del estilo de vida saludable, los estudiantes forman también su cultura física personal (Meleshkova et al., 2014).

El sistema moderno de la educación física de estudiantes de las facultades que no se refieren a la formación física es dominante en el desarrollo de la cultura física y deporte como componentes estructurales de la cultura humana general, el estilo de vida saludable y la competencia profesional del especialista. La cultura física no es solo un medio de entrenamiento físico, preservación y fortalecimiento de la salud física, sino también el mecanismo de interacción interpersonal (Bugulov \& Sokaev, 2018); la condición de cooperación social exitosa (Meleshkova et al., 2014); la formación de orientaciones valorativas que contribuyen al mejoramiento consciente de sus cualidades físicas, morales y personales (Vnukov, 2014).
El estilo de vida saludable y el entrenamiento físico son una parte integral de la formación de la cultura general y profesional del especialista (Morosanova \& Konoz, 2000). La cultura física establece las pautas básicas de libre autodeterminación del individuo, que, en diferentes periodos de la actividad vital, de todo el conjunto de valores elige los que son por el momento los más importantes.

De interés científico son los estudios en los que los estudiantes que creen en la importancia del estilo de vida saludable, de la actividad física como uno de los principales valores personales, son capaces de organizar y mantener el estilo de vida saludable. Estos encuestados demuestran independencia, determinación y perseverancia en muchas esferas de su vida después de terminar la carrera también. El alto nivel de formación de la orientación personal y la motivación de los estudiantes hacia la actividad deportiva sistemática aumenta significativamente la eficacia de sus cualidades físicas así que las cualidades profesionales importantes. Como resultado, ellos demuestran la actitud valorativa hacia el estilo de vida saludable (Tóth et al., 2019).

Para que el estudiante logre mejores resultados en futuras actividades profesionales, es necesario que motivarlo a fin de que tenga ganas de conseguir éxito. El estilo de vida saludable, la educación física y los deportes pueden servir de buen medio de motivación de los estudiantes hacia el éxito. La necesidad formada de tener éxito desarrolla a un joven la alta actividad, la perseverancia para alcanzar el objetivo, la motivación del éxito ayuda a superar diversos obstáculos. Hay estudios en los que se demuestra que los encuestados que llevan la vida sana, practican la cultura física y el deporte, tienen altos índices del éxito, son capaces de establecer objetivos conscientes y se esfuerzan no solo para lograr altos resultados, sino que también tienen el sentido desarrollado de deber y de responsabilidad (Kovacs, 2019; Kubieva et al., 2019).

Hipótesis. Los autores suponen que los estudiantes que llevan una vida sana y piensan que las clases de cultura física son un valor personal, son más capaces a la autorregulación voluntaria en la actividad y están más orientados a lograr el éxito en la vida.

Propósito. Determinar la actitud valorativa de la juventud estudiantil hacia el estilo de vida saludable, la cultura física y el impacto a la manifestación de las cualidades de 


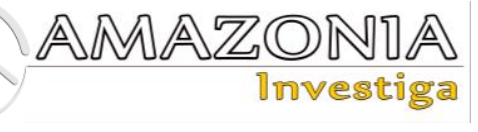

autorregulación volitiva del comportamiento y el deseo de triunfar.

\section{Método}

Los puntos de partida metodológicos del estudio empírico de la actitud valorativa de la juventud estudiantil hacia el estilo de vida saludable y la actividad física como factores de su deseo de tener éxito son un conjunto de medidas sucesivas con el uso de herramientas de psicodiagnóstico. Esta metodología fue aprobada por los investigadores al estudiar el espacio educativo seguro y la motivación de logro de los encuestados (Blynova et al., 2020d; Popovych et al., 2020c), los procesos de adaptación (Blynova et al., 2020a; 2020b; 2020c), la autorregulación del comportamiento de los encuestados (Khmiliar et al., 2020; Kuzikova et al., 2020), la investigación de la formación educativa $y$ profesional de futuros profesionales (Halian et al., 2020; Popovych et al., 2020a; 2020b ; 2021), así que en la investigación de otras actividades humanas (Arbeláez-Campillo et al., 2019; 2020; Nosov et al., 2020a; 2020b; Shevchenko et al., 2020; Villasmil Espinoza et al., 2021; Zinchenko et al., 2019; 2020). Todos los estudios considerados experimentales y empíricos contenían elementos de la actitud valorativa, autorregulación volitiva, aspectos motivacionales de la aspiración de éxito de los encuestados.

Participantes. En el estudio participaron 124 estudiantes de 1 a 4 cursos de estudios presenciales de la Academia Interregional de Gestión de Personal (Kiev, Ucrania), además de los estudiantes de la Facultad de Cultura Física y Deportes.

Procedimiento e instrumentos. La evaluación de las actitudes de estudiantes hacia los valores del estilo de vida saludable y cultura física fue realizado con ayuda del cuestionario de autor elaborado de antemano. El cuestionario refleja el nivel de cobertura de los estudiantes por diversas formas de estilo de vida saludable, ejercicios físicos, el círculo de sus intereses físicos deportivos, necesidades, motivos. La característica cuantitativa cualitativa de los parámetros del estilo de vida saludable, de la actividad física está representada por los indicadores del tiempo dedicado a la actividad física, el descanso activo, la regularidad de ejercicios físicos y la motivación de los estudiantes hacia la cultura física.

Para estudiar la esfera volitiva de los estudiantes fue utilizado el cuestionario "Estilo de autorregulación del comportamiento" (Morosanova \& Konoz, 2000). Las afirmaciones del cuestionario se basan en las situaciones típicas de la vida y no están directamente relacionadas con las particularidades de la actividad profesional o educativa. El cuestionario tiene la escala "Nivel general de autorregulación" (NG), que caracteriza el nivel de formación del sistema individual de autorregulación de la actividad humana arbitraria. Además, existen seis escalas precisadas de acuerdo con los principales procesos reguladores: planificación (Pl), modelización (M), programación (Pr), evaluación de resultados (Er); así que las propiedades reguladores personales: flexibilidad (F) e independencia (I). Algunas afirmaciones están incluidas en dos escalas a la vez.

Para diagnosticar la necesidad de conseguir el éxito, los objetivos, la motivación de logro se utilizó el test-cuestionario "Necesidad de logro" (Orlov, 1998). La motivación de logro se manifiesta en el deseo de mejorar los resultados, la perseverancia para alcanzar sus objetivos, la necesidad de superar obstáculos, la superación personal (competir con otras personas y sobrepasarlas), el deseo de autorrealización, elevación del nivel de autoestima.

Análisis estadístico. El procesamiento estadístico de los datos empíricos se realizó mediante los programas estadísticos "SPSS" v. 25.0. Para calcular los coeficientes de correlación se utilizó el coeficiente de correlación de Spearman. La diferencia estadística entre los grupos se estableció mediante el criterio $\varphi$ de la transformación angular de Fisher.

\section{Resultados y discusión}

Según el cuestionario del parámetro de tiempo dedicado al estilo de vida saludable, la actividad física, los estudiantes fueron divididos en tres grupos: 1) Los estudiantes que no demuestran actividad física, es decir, hasta 1 hora por semana $(\mathrm{n}=36 ; 29.04 \%)$. Para este grupo de estudiantes, la educación física no se ha convertido en una necesidad vital, no tienen motivos en esta esfera de actividad; 2) Los estudiantes con cultura física y actividad deportiva óptimas: hasta 6 horas por semana $(\mathrm{n}=58 ; 46.77 \%)$. El motivo de este grupo de estudiantes es la necesidad de cumplir con los estándares del plan de estudios y obtener "aprobado"; la cantidad real de actividad física no asegura el pleno desarrollo y promoción de la salud; 3) Los estudiantes con actividad física 
deportiva relativamente alta: 7-8 o más horas por semana $(n=30 ; 24.19 \%)$.

$\mathrm{Al}$ analizar las respuestas de los estudiantes a las cuestiones sobre la suficiencia del régimen dinámico para la vida y la salud normales, el $34.21 \%$ de los encuestados dio la respuesta satisfactoria, el $30.70 \%$ escogió "es difícil responder"; el 35.09\% está dispuesto admitir que su propio régimen dinámico es insuficiente.

Las características descriptivas de los resultados de la metodología "Estilo de autorregulación del comportamiento" (Morosanova \& Konoz, 2000) están presentadas en la Tabla 1.

Tabla 1.

Las características descriptivas según las escalas del método "Estilo de autorregulación del comportamiento" $(n=124)$

\begin{tabular}{lll}
\hline Escala & M & SD \\
\hline Planificación (Pl) & 5.68 & 2.10 \\
Modelado (M) & 6.12 & 1.78 \\
Programación (Pr) & 4.89 & 2.09 \\
Evaluación de resultados (Er) & 6.31 & 1.81 \\
Flexibilidad (F) & 5.80 & 2.12 \\
Independencia (I) & 4.71 & 1.55 \\
Escala general de autorregulación & 29.39 & 4.33 \\
\hline
\end{tabular}

Nota: M - media aritmética; SD - desviación de la media cuadrada.

Fuente: Elaboración propia, 2021.

Al comparar los datos obtenidos con los indicadores normativos según las escalas de la metodología, se determinó que según las escalas "Planificación" $(\mathrm{M}=5.68 ; \mathrm{DT}=2.10)$; "Programación" $(\mathrm{M}=4.89 ; \mathrm{SD}=2.09)$; "Flexibilidad" $(\mathrm{M}=5.80 ; \mathrm{DT}=2.12)$ fue obtenido el nivel promedio de las características correspondientes. Las escalas "Modelado" $(\mathrm{M}=6.12 ; \mathrm{DT}=1.78) \mathrm{y}$ "Evaluación de resultados" $(\mathrm{M}=6.31$; DT $=1.81)$ mostraron el nivel superior al promedio, lo que indica la capacidad de los estudiantes de identificar condiciones significativas para alcanzar un objetivo tanto en la situación actual como en futuro. Además, la muestra de estudiantes se caracteriza por la formación y constancia de criterios subjetivos de la evaluación de resultados. También cabe señalar un descenso leve de indicadores en comparación con las evaluaciones normativas de la escala "Independencia" $(\mathrm{M}=4,71$; DT $=1,55)$, es decir, en ocasiones los planes y programas de comportamiento se construyen bajo la influencia de situaciones, circunstancias, otras personas.

En general, la mayoría de las voloraciones medias obtenidas en toda la muestra de investigación incitan a hacer un análisis más diferenciado.

De acuerdo con los resultados del testcuestionario "Necesidad de logro" (Orlov, 1998) se obtuvó siguientes valores medios de grupo $(\mathrm{M}=14,67 ; \mathrm{DE}=3,27)$, que al compararlos con la escala de normas de test demuestra que la necesidad de logro está por encima de la media. Las personas con alto nivel de necesidad de logro tienen siguientes características: la insatisfacción con lo logrado, el deseo de hacerlo mejor que antes, la tendencia a disfrutar de las actividades, el deseo de experimentar el placer del éxito, la necesidad de encontrar nuevos herramientas de trabajo; ellas están dispuestas aceptar ayuda y ayudar a otros a resolver problemas difíciles para disfrutar juntos de la alegría del éxito.

Para hacer el análisis de correlación, el indicador del nivel de cultura física y actividad deportiva se transformó en la escala de intervalo y se marcó de manera siguiente: nivel bajo - 1 punto; nivel medio - 2 puntos; por encima del nivel medio 3 puntos en la Tabla 2. 
Tabla 2.

Las relaciones de correlacion según las escalas de la metodología "Estilo de autorregulación del comportamiento" (Morosanova \& Konoz, 2000), los indicadores "Necesidad de logro" (Orlov, 1998) y el nivel de actividad física.

\begin{tabular}{|c|c|c|}
\hline $\begin{array}{l}\text { Criterios de autorregulación del } \\
\text { comportamiento }\end{array}$ & Necesidad de logro & $\begin{array}{l}\text { Nivel de actividad } \\
\text { deportiva }\end{array}$ \\
\hline Planificación (Pl) & .268 *** & $.229^{*}$ \\
\hline Modelado (M) & .179 & 159 \\
\hline Programación (Pr) & .189 & .187 \\
\hline Evaluación de resultados (Er) & $.259^{* * *}$ & .168 \\
\hline Flexibilidad (F) & $.309^{* *}$ & .179 \\
\hline Independencia (I) & $.331^{* *}$ & $.254^{* *}$ \\
\hline
\end{tabular}

Nota: ${ }^{*}-\mathrm{p} \leq .05 ;{ }^{* *}-\mathrm{p} \leq .01$.

Fuente: Elaboración propia, 2021.

El análisis de correlación mostró la presencia de relaciones estadísticamente significativas entre la necesidad de logro de la juventud estudiantil y los parámetros de autorregulación del comportamiento propio, es decir, según las escalas: "Planificación" $\left(\mathrm{r}_{\mathrm{s}}=.268 ; \mathrm{p}<.01\right)$; "Evaluación de resultados" $\left(\mathrm{r}_{\mathrm{s}}=.259 ; \mathrm{p}<.01\right)$; "Flexibilidad" $\quad\left(\mathrm{r}_{\mathrm{s}}=.309 ; \quad \mathrm{p}<.01\right)$; "Independencia" $\quad\left(\mathrm{r}_{\mathrm{s}}=.331 ; \mathrm{p}<.01\right)$. Estos resultados confirman que la persona que tiene los altos indicadores de necesidad de logro, es capaz de planificar conscientemente sus actividades, construir planes realistas y detallados, ser independiente en la formulación de objetivos. Además, el alto nivel de necesidad de logro determina la adecuación y constancia de los criterios subjetivos de la evaluación de los resultados permitiendo adaptarse con flexibilidad a las condiciones cambiantes. Además, la necesidad de éxito está caracterizada por alto nivel de autonomía, es decir, la capacidad de planificar actividades y comportamientos de forma independiente, organizar trabajo para alcanzar objetivos, controlar el cumplimiento del plan, analizar y evaluar los resultados intermedios y finales de la actividad.

El estilo de vida saludable, el alto nivel de cultura física y la actividad deportiva, según nuestra hipótesis, están determinados por las características volitivas del individuo, es decir, por los parámetros de autorregulación. De acuerdo con los resultados del análisis de correlación, se estableció sólo dos interrelaciones estadísticamente significativas: con la escala "Planificación" ( $\left.\mathrm{r}_{\mathrm{s}}=.229 ; \mathrm{p}<.05\right)$ y la escala "Independencia" $\left(\mathrm{r}_{\mathrm{s}}=.254 ; \mathrm{p}<.01\right)$, es decir que los estudiantes que tienen el nivel más alto de actividad física independiente, se caracterizan por la capacidad de planificar y organizar de forma independiente sus actividades, una cierta disciplina y coherencia de sus actividades actuales con el objetivo final.

Sin embargo, estos resultados del análisis de correlación indican al mismo tiempo que existe la relación no lineal entre el nivel de actividad física, que es el reflejo de la actitud de valor hacia la cultura física, y las cualidades que determinan la regulación del comportamiento. Esta suposición estimuló a realizar los estudios posteriores.

En la siguiente etapa de nuestros estudios, se destacaron dos subgrupos polares de la muestra de investigación para hacer el análisis comparativo: grupo 1 - los estudiantes que no demuestran cultura física y actividad deportiva ( $\mathrm{n}$ = 36; 29,04\%); grupo $2-$ los estudiantes con cultura física y actividad deportiva relativamente altas $(\mathrm{n}=30 ; 24,19 \%)$.

Para comprobar la diferencia estadística entre dos muestras se utilizó el criterio de transformación angular de Fisher en la Tabla 3. Para hacer la comparación tomaron el porcentaje de personas de cada subgrupo que tienen el alto nivel del indicador estudiado. 
Tabla 3.

Las diferencias entre los grupos según los criterios de autorregulación del comportamiento $\left(n_{1}=36 ; n_{2}=30\right)$

\begin{tabular}{lllll}
\hline Escala & $\begin{array}{l}\text { Grupo 1 } \\
\left(\mathbf{n}_{\mathbf{1}}=\mathbf{3 6}\right), \boldsymbol{\%}\end{array}$ & $\begin{array}{l}\text { Grupo 2 } \\
\left(\mathbf{n}_{\mathbf{2}}=\mathbf{3 0}\right) \mathbf{\%}\end{array}$ & $\begin{array}{l}\text { Criterio de } \\
\text { Fisher, } \boldsymbol{\varphi} \text { emp. }\end{array}$ & Valor crítico \\
\hline Planificación (Pl) & 14.8 & 37.0 & 1.89 & $\mathrm{p} \leq .05$ \\
Modelado (M) & 14.8 & 26.1 & - & - \\
Programación (Pr) & 23.6 & 26.1 & - & - \\
Evaluación de & 17.9 & 44.2 & 2.23 & $\mathrm{p} \leq .05$ \\
resultados (Er) & 11.8 & 33.5 & 2.01 & $\mathrm{p} \leq .05$ \\
Flexibilidad (F) & 20.7 & 40.8 & 1.71 & $\mathrm{p} \leq .05$ \\
Independencia (I) & &
\end{tabular}

Fuente: Elaboración propia, 2021.

Se estableció las diferencias entre el grupo 1 y el grupo 2 en algunos parámetros. El grupo 1 (con bajo nivel de actividad física) presenta indicadores más bajos en comparación con el grupo 2 (con alto nivel de actividad física) según el criterio "Planificación" ( $\left.\varphi_{\text {emp. }}=1,89 ; \mathrm{p}<.05\right)$; en el grupo 2 el criterio de autorregulación del comportamiento "Evaluación de resultados" $\left(\varphi_{\text {emp. }}=2.23 ; \mathrm{p}<.05\right)$ está más manifestado que demuestra que la conciencia de los estudiantes de la cultura física como valor motiva planificar mejor, realizar y evaluar sus resultados, y eso se refiere no solo a las actividades deportivas. También existen diferencias estadísticamente significativas entre el grupo 1 y el grupo 2 según las características siguientes de autorregulación del comportamiento "Flexibilidad" $\left(\varphi_{\text {emp. }}=2.01\right.$; $p<.05)$ e "Independencia" $\left(\varphi_{\text {emp. }}=1.71 ; p<.05\right)$, es decir, los estudiantes que practican la cultura física y las actividades deportivas son más capaces de adaptarse a las circunstancias cambiantes, son capaces de reestructurar adecuadamente el programa de acción teniendo en cuenta todas las situaciones cambiantes, demuestran la capacidad de responder adecuadamente y resolver problemas de manera oportuna. Los criterios de autorregulación del comportamiento de tres grupos estudiados están presentados gráficaminete en la Fig. 1.

기

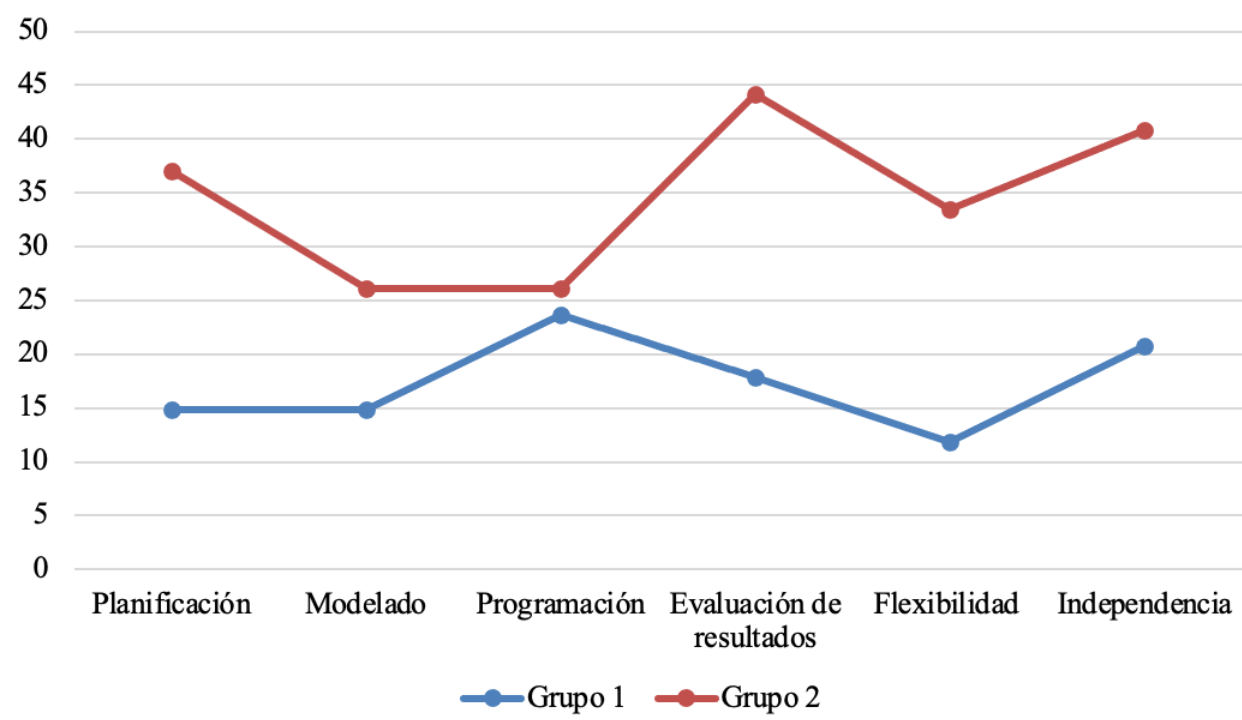

Figura 1. El diagrama de indicadores de criterios de autorregulación voluntaria de los encuestados del Grupo 1 y Grupo 2.

Fuente: Elaboración propia, 2021.

Los indicadores más bajos de los parámetros "Evaluación de resultados" e "Independencia" se registraron en el Grupo 3 a diferencia del Grupo 2. Esto lo explicamos por el hecho que el nivel de actividad física y deportiva superior al promedio (Grupo 3) se acompaña por la dependencia no lineal de estos parámetros estudiados lo que necesita ser investigado más. Es obvio que estos indicadores están relacionados en gran medida con el desarrollo de los procesos cognitivos y son menos dependientes de la regulación volitiva del comportamiento de los encuestados. 


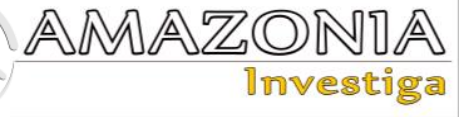

Se determinó que las escalas de la metodología "Estilo de regulación del comportamiento" (Morosanova \& Konoz, 2000) "Programación" y "Modelado" no mostraron correlaciones estadísticamente significativas con el nivel de actividad física. Esto se explica por el hecho que la capacidad de identificar las condiciones para alcanzar objetivos en el futuro, detallar, pensar, ajustar planes y programar el comportamiento, se debe principalmente a procesos cognitivos complejos. Pero está menos relacionado con los componentes de valor de la regulación consciente del comportamiento.

\section{Conclusiones}

1. Constatamos la importante interdependencia entre el estilo de vida saludable, el nivel de actividad física de los estudiantes y su deseo de tener éxito ( $\mathrm{p}<.01)$.

2. Se estableció una relación estadísticamente significativa entre los parámetros de autorregulación del comportamiento propio, a saber "Planificación", "Evaluación de resultados", "Flexibilidad", "Independencia" y la necesidad de tener éxito de la juventud estudiantil ( $\mathrm{p}<.01)$.

3. Se determinó las diferencias entre los grupos de estudiantes con niveles altos y bajos de actividad física según los parámetros de autorregulación del comportamiento - "Planificación" ( $p$ $<.05)$, "Evaluación de resultados" ( $<.05)$, "Flexibilidad" (p <.05), "Independencia" ( $p<.05$ ), lo que demuestra que la conciencia de la cultura física como valor afecta las cualidades volitivas de la personalidad de los estudiantes y el nivel de su necesidad de lograr éxito.

4. Los resultados obtenidos dan fundamento a la conclusión de que el estilo de vida saludable de los estudiantes debe realizarse como formación de una actitud consciente y activa hacia la asimilación de los valores de la cultura física. Durante el período de estudio en la universidad, los estudiantes deben tener confianza en la necesidad de trabajar constantemente en sí mismos, llevar una vida sana, estudiar las particularidades de su organismo. Tienen que aprender a utilizar racionalmente su potencial físico para llevar la vida sana.

5. La actitud de los estudiantes hacia la cultura física como valor es un factor efectivo de la formación del estilo de vida saludable, habilidades intelectuales, de "Yo", así que cualidades profesionales importantes.

\section{Referencias bibliográficas}

Arbeláez-Campillo, D., \& Rojas-Bahamón, M., et al. (2019). Apuntes para el debate de las categorías ciudadanía universal, derechos humanos y globalización. Notes for the debate of the categories universal citizenship, human rights and globalization. Cuestiones Políticas, 34(61), 139-161. https://produccioncientificaluz.org/index.php /cuestiones/article/view/30192

Arbeláez-Campillo, D., Tatsiy, V., Rojas-Bahamón, M., \& Danilyan, O. (2020). Contributions of critical thinking as a form of participation and political deliberation. Amazonia Investiga, 9(27), 5-12. https://doi.org/10.34069/AI/2020.27.03.1

Blynova, O., Chervinska, I., Kazibekova, V., et al. (2020a). Social and Psychological Manifestations of Professional Identity Crisis of Labor Migrants. Revista Inclusiones, 7(3), 93-105.

http://www.revistainclusiones.org/index.php /inclu/article/view/1318

Blynova, O., Kisil, Z., Tkach, T., et al. (2020b). Psychological manifestations of professional marginality of future social welfare professionals. Revista Inclusiones, 7(SI), 218-233.

http://www.revistainclusiones.org/index.php /inclu/article/view/1229

Blynova, O., Moiseienko, V., Los, O., et al. (2020c). Assertiveness as a Factor of Students' Choice of Behavior Strategies in Social Interaction. Revista Inclusiones, 7(4), 259-272.

http://www.revistainclusiones.org/index.php /inclu/article/view/1551

Blynova, O., Popovych, I., Semenova, N., Kashyrina, Y., Ursulenko, O., \& Kononenko, O. (2020d). Personality Factors of Choosing Adaptation Strategies in a Different Cultural Environment by Labor Migrants from Ukraine. Revista Amazonia Investiga, $\quad 9(32), \quad$ 45-54. http://dx.doi.org/10.34069/AI/2020.32.08.5

Bugulov, A. G. \& Sokaev, H. M. (2018). The role of physical education in the formation of practical human competence. News of Science and Education, 3(3), 33-36. https://elibrary.ru/item.asp?id=32535631

Burcev, V. A., Burceva, E. V. \& Martynova, A. S. (2014). Criteria, indicators and methods for measuring the level of 
development of a sports culture of an individual. Basic research, 11, 1147-1151. https://fundamentalresearch.ru/ru/article/view?id=35694

Byhovskaja, I. M. (2011). Sport: cultural vectors of the analysis of the phenomenon. Journal of Cultural Research, 1. https://cyberleninka.ru/article/n/sportkulturologicheskie-vektory-analizafenomena/viewer

Halian, I., Halian, O., Gusak, L., Bokshan, H., \& Popovych, I. (2020). Communicative Competence in Training Future Language and Literature Teachers. Revista Amazonia Investiga, 9(29), 530-541. https://amazoniainvestiga.info/index.php/am azonia/article/view/1417

Khmiliar, O., Popovych, I., Hrys, A., et al. (2020). Spatial Regulation of Personality Behavior in the Conditions of Progression of the COVID-19 Pandemic. Revista Inclusiones, 7(Especial), 289-306. http://www.revistainclusiones.org/index.php /inclu/article/view/1760

Kovacs, K. (2019). Socio-cultural characteristics of sport activity among students in Central and Eastern Europe: Comparative empirical analysis. Journal of Physical Education and Sport, 19(1), 669-676. https://efsupit.ro/images/stories/April2019/A rt96.pdf

Kubieva, S., Botagariev, T., Konisbaeva, S., et al. (2019). Health promotion program for the students with regard to the level of their physical activity, physical fitness and health. Journal of Physical Education and Sport, 19(1), 703-709. https://efsupit.ro/images/stories/April2019/A rt101.pdf

Kuzikova, S., Shcherbak, T., Popovych, I., et al. (2020). Psychological features of subjective vitality and hardiness of representatives of parachute sports. International Journal of Applied Exercise Physiology, 9(9), 172-177. http://ekhsuir.kspu.edu/123456789/12265

Meleshkova, N., Grigorieva, S. \& Bukreyeva, N. (2016). Physical education and the formation of a culture of health and wellness in university students. Professional Education in Russia and Abroad, 1(21), 161-164. https://cyberleninka.ru/article/n/fizicheskoevospitanie-i-formirovanie-kultury-zdorovyazdorovogo-obraza-zhizni-u-studentovvuza/viewer

Morosanova, V. I. \& Konoz, E. M. (2000). Style self-regulation of human behavior. Psychology Issues, 2, 118-127. https://elibrary.ru/item.asp?id=37337299
Nosov, P., Palamarchuk, I., Zinchenko, S., et al. (2020a). Development of means for experimental identification of navigator attention in ergatic systems of maritime transport. Bulletin of the University of Karaganda - Physics, 1(97), 58-69. https://physics-vestnik.ksu.kz/apart/2020-971/7.pdf

Nosov, P. S., Zinchenko, S. M., Popovych, I. S. et al. (2020b). Diagnostic system of perception of navigation danger when implementation complicated maneuvers. Radio Electronics, Computer Science, Control, 1, 146-161. https://doi.org/10.15588/1607-3274-2020-115

Orlov, Ju. M. (1998). Methods for identifying the need for reach. St. Petersburg: Peter. https://infopedia.su/16x267b.html

Popovych, I., Arbeláez-Campillo, D., Rojas-Bahamón, M., et al. (2021). Time perspective in the professional activity of specialists of economic sphere. Cuestiones Políticas, 39(69), 424-445. https://doi.org/10.46398/cuestpol.3969.27

Popovych, I., Halian, O., Bokhonkova, Yu., et al. (2020a). Research of the Motivational Mental States of Future Bachelors of the Faculty of Physical Education and Sports in the Educational Process. Revista Inclusiones, 7(4), 159-178. http://www.revistainclusiones.org/index.php /inclu/article/view/1545

Popovych, I., Halian, O., Geiko, Ie., et al. (2020b). Research on the Correlation between Subjective Control and the Indexes of Study Progress of Bachelors of the Faculty of Physical Education and Sport. Revista Inclusiones, 7(SI), 497-511. http://www.revistainclusiones.org/index.php /inclu/article/view/1545

Popovych, I., Zhigarenko, I., Losiyevska, O., et al. (2020c). Research of Achievement Motivation's Impaction the Career Orientations of Future Managers of Organization. Revista Inclusiones, 7(SI), 247-263.

http://www.revistainclusiones.org/index.php /inclu/article/view/1231

Shevchenko, R., Cherniavskyi, V., Zinchenko, S., et al. (2020). Research of psychophysiological features of response to stress situations by future sailors. Revista Inclusiones, 7(SI), 566-579. http://www.revistainclusiones.org/index.php /inclu/article/view/1780

Tóth, L., Lőkös, D., Sipos, K., et al. (2019). Exercise involvement and trait-anxiety are determinants of physical self-concept: 


\section{AMAZOND周 \\ lnvestiga}

exercisers exhibit superior profiles compared with non-exercisers. Journal of Physical Education and Sport, 19(1), 580-585. https://efsupit.ro/images/stories/April2019/A rt85.pdf

Villasmil Espinoza, J., Arbeláez-Campillo, D., Rojas-Bahamón, M., et al. (2021). ¿Crisis del liderazgo político o liderazgo en escenarios de crisis? Revista Notas Históricas y Geográficas, 26, 269-292. https://revistanotashistoricasygeograficas.cl/ carga/wp-content/uploads/2021/04/10-

Villasmil-et-al-Notas-1-2021.pdf

Vnukov, A. V. (2014). Value orientations of student personality as regulators of social behavior. Bulletin of the University (State University of Management), 1, 237-239.

https://cyberleninka.ru/article/n/tsennostnyeorientatsii-lichnosti-studenta-kakregulyatory-sotsialnogo-povedeniya/viewer

Zinchenko, S. M., Ben, A. P., Nosov, P. S., et al. (2020). IMPROVING THE ACCURACY AND RELIABILITY OF AUTOMATIC VESSEL MOUTION CONTROL SYSTEM. Radio Electronics, Computer Science, Control, 2, 183-195. https://doi.org/10.15588/1607-3274-2020-219

Zinchenko, S., Nosov, P., Mateichuk, V., et al. (2019). Automatic collision avoidance system with many targets, including maneuvering ones. Bulletin of university of Karaganda, 96(4), 69-79. https://physicsvestnik.ksu.kz/apart/2019-96-4/8.pdf 